\title{
Calorimetric and relaxation properties of xylitol-water mixtures
}

\author{
Khalid Elamin, Johan Sjöström, ${ }^{\text {a) }}$ Helén Jansson, ${ }^{\text {b) }}$ and Jan Swenson ${ }^{c}$ ) \\ Department of Applied Physics, Chalmers University of Technology, SE-41296 Göteborg, Sweden
}

(Received 8 November 2011; accepted 18 February 2012; published online 14 March 2012)

\begin{abstract}
We present the first broadband dielectric spectroscopy (BDS) and differential scanning calorimetry study of supercooled xylitol-water mixtures in the whole concentration range and in wide frequency $\left(10^{-2}-10^{6} \mathrm{~Hz}\right)$ and temperature $(120-365 \mathrm{~K})$ ranges. The calorimetric glass transition, $\mathrm{T}_{\mathrm{g}}$, decreases from $247 \mathrm{~K}$ for pure xylitol to about $181 \mathrm{~K}$ at a water concentration of approximately $37 \mathrm{wt} . \%$. At water concentrations in the range $29-35 \mathrm{wt}$. \% a plentiful calorimetric behaviour is observed. In addition to the glass transition, almost simultaneous crystallization and melting events occurring around $230-240 \mathrm{~K}$. At higher water concentrations ice is formed during cooling and the glass transition temperature increases to a steady value of about $200 \mathrm{~K}$ for all higher water concentrations. This $\mathrm{T}_{\mathrm{g}}$ corresponds to an unfrozen xylitol-water solution containing $20 \mathrm{wt}$. \% water. In addition to the true glass transition we also observed a glass transition-like feature at $220 \mathrm{~K}$ for all the ice containing samples. However, this feature is more likely due to ice dissolution [A. Inaba and O. Andersson, Thermochim. Acta, 461, 44 (2007)]. In the case of the BDS measurements the presence of water clearly has an effect on both the cooperative $\alpha$-relaxation and the secondary $\beta$-relaxation. The $\alpha$-relaxation shows a non-Arrhenius temperature dependence and becomes faster with increasing concentration of water. The fragility of the solutions, determined by the temperature dependence of the $\alpha$-relaxation close to the dynamic glass transition, decreases with increasing water content up to about $26 \mathrm{wt}$ \% water, where ice starts to form. This decrease in fragility with increasing water content is most likely caused by the increasing density of hydrogen bonds, forming a network-like structure in the deeply supercooled regime. The intensity of the secondary $\beta$-relaxation of xylitol decreases noticeably already at a water content of $2 \mathrm{wt} . \%$, and at a water content above $5 \mathrm{wt}$. \% it has been replaced by a considerably stronger water (w) relaxation at about the same frequency. However, the similarities in time scale and activation energy between the w-relaxation and the $\beta$-relaxation of xylitol at water contents below $13 \mathrm{wt}$. \% suggest that the w-relaxation is governed, in some way, by the $\beta$-relaxation of xylitol, since clusters of water molecules are rare at these water concentrations. At higher water concentrations the intensity and relaxation rate of the w-relaxation increase rapidly with increasing water content (up to the concentration where ice starts to form), most likely due to a rapid increase of small water clusters where an increasing number of water molecules interacting with other water molecules. () 2012 American Institute of Physics. [http://dx.doi.org/10.1063/1.3692609]
\end{abstract}

\section{INTRODUCTION}

Water covers almost two thirds of the surface of our planet and it is the most important substance on earth, without which life, as we know it, would not be possible. Water exhibits also many peculiar properties, such as unusually high surface tension and heat capacity, and a density maximum at a temperature of $4 \mathrm{~K}$ above its melting temperature. Both these peculiar properties of water and its related biological importance are due to the hydrogen bonds between the molecules. Thus, hydrogen bonds play an essential role not only for the structural and dynamical properties of water but also for the properties of other hydrogen bonded liquids as well as aqueous solutions and mixtures of hydrogen bonded liquids. However, despite intensive research in recent years on

\footnotetext{
a) Present address: SP Technical research Institute of Sweden, SE-501 15 Borås, Sweden.

b) Present address: Department of Civil and Environmental Engineering, Chalmers University of Technology, SE-412 96 Göteborg, Sweden.

${ }^{c)}$ Author to whom correspondence should be addressed. Electronic mail: jan.swenson@chalmers.se.
}

hydrogen bonded liquids the detailed role of hydrogen bonds is still only poorly understood and therefore further structural and dynamic studies are needed to clarify the many mysterious properties of these intermolecular interactions. Both pure water and different types of aqueous mixtures are important model systems to understand the physical and chemical nature of hydrogen bonds.

One such model system is xylitol mixed with water. This system has, furthermore, many applications in food materials, such as chewing gum, mints, jellies, and chocolate, ${ }^{1,2}$ as well as in hygienic products such as toothpastes and mouthwashes. ${ }^{3,4}$ These applications have stimulated a few studies of xylitol-water mixtures in the supercooled state, with the aim to elucidate how the $\alpha$ - and $\beta$-relaxations of xylitol are affected by the introduction of water. ${ }^{5,6}$ In anhydrous materials the secondary $\beta$-relaxation process is of Johari-Goldstein character. ${ }^{7}$ When water is added to the material this $\beta$-relaxation becomes difficult to observe due to the presence of a water (w) relaxation in the same frequency range. ${ }^{8-14}$ In Ref. 6 the authors observed that the $\alpha$-relaxation speeds up with increasing concentration of 
water, while the Johari-Goldstein $\beta$-relaxation remained practically unchanged. There has also been similar studies on other aqueous mixtures, such as water mixed with sorbitol, ${ }^{5,15}$ glycerol,${ }^{8,16}$ fructose,${ }^{17}$ glucose, fructose and sucrose, ${ }^{18}$ and n-propylene glycol. ${ }^{9}$ In all these studies the addition of water to the glass-forming material gives rise to a specific water relaxation in the glassy state of the solution. However, this wrelaxation, due to reorientational motions of water molecules, has shown slightly different behaviours in the different systems and the water has also had different effects on the glass transition related $\alpha$-relaxation. ${ }^{10}$ In Ref. 10 it was observed that when water was mixed with propylene glycol (PG), having two hydroxyl $(\mathrm{OH})$ end-groups, both the $\alpha$ - and the wrelaxation became faster with increasing water concentration at temperatures near $\mathrm{T}_{\mathrm{g}}$, but an opposite behaviour was observed for the $\alpha$-relaxation in propylene glycol monomethyl ethers (n-PGME), having only one $\mathrm{OH}$ end-group. This slowing down of the $\alpha$-relaxation in n-PGME with increasing water content (up to a certain water concentration) can be explained in terms of a water induced grows of effective relaxing entities. ${ }^{19}$ In pure n-PGME the molecules tend to form pairs connected with their $\mathrm{OH}$ end-groups, ${ }^{20}$ and these pairs of molecules relax as single entities. However, when water is added the water molecules have a larger tendency to interact with the $\mathrm{OH}$ end-groups of the n-PGME molecules, thereby forming bridges between two n-PGME molecules. This increases the size of the effective relaxing entities, which, in turn, slows down the $\alpha$-relaxation and increases the related glass transition temperature. ${ }^{19,21}$

In this study we have used differential scanning calorimetry (DSC) and broadband dielectric spectroscopy (BDS) to study the calorimetric and relaxation behaviours of xylitol and xylitol-water mixtures in the broad temperature range 120-365 K. As far as we are aware of, this is the first such study over the whole concentration range, and this has led to that we have observed new calorimetric and relaxation behaviours in the supercooled regime. Thus, compared to the previous xylitol-water studies ${ }^{5,6}$ we have gone to considerably higher water concentrations (only water concentrations up to 10 wt. \% was studied with BDS in Ref. 5) and have therefore been able to study the concentration behaviour of the w-relaxation in more detail, as well as investigating what is happening at high water contents when the solution becomes partially crystalline. At such high water concentrations our DSC studies show a complex calorimetric behaviour similar to what previously ${ }^{16}$ have been obtained for the related glycerol-water system. At high water concentrations, where the solution is partially crystalline (i.e., ice particles have been formed), we observe two glass-transition-like features. However, in analogy to the interpretations made in Ref. 16 only the feature at the lower temperature is due to a true glass transition, whereas the other feature is due to dissolution of ice with increasing temperature. Furthermore, at moderate and high water concentrations the BDS data show that the secondary dielectric relaxation, caused by water, is strongly dependent on the water concentration. This finding is in contrast to what could be concluded from the limited concentration range studied in Ref. 5, but in agreement to what has been found for other aqueous mixtures, such as n-propylene glycol,${ }^{9,10}$ ribose, ${ }^{22}$ and deoxyribose ${ }^{22}$ mixed with water. The reason for the rapidly increase of its relaxation rate and intensity with increasing water concentration is discussed.

\section{MATERIALS AND METHODS}

We have investigated mixtures of xylitol $\left(\mathrm{C}_{5} \mathrm{H}_{12} \mathrm{O}_{5}\right)$ ( $\geq 99 \%$ purity, purchased from Sigma, C.A.S number (87-99$0)$ ) and double distilled water (Milli-Q water) over a broad concentration range from pure xylitol up to $80 \mathrm{wt}$. \% water. The boiling, melting, and glass transition temperature for pure xylitol are $489 \mathrm{~K}, 367 \mathrm{~K}$, and $247 \mathrm{~K}$, respectively. ${ }^{23,24}$ Dielectric measurements were performed on a broadband dielectric spectrometer from Novocontrol, equipped with a Novocontrol Alfa-S High Resolution Dielectric Analyser. The measurements were performed in the frequency and temperature ranges $10^{-2}-10^{6} \mathrm{~Hz}$ and $120-365 \mathrm{~K}$, respectively. The temperature was controlled using a nitrogen gas cryostat, with temperature stabilization better than $\pm 0.2 \mathrm{~K}$. Frequency scans were made at every fifth degree. Before each measurement the sample was ultrasonificated long enough (several minutes) to get a homogenous liquid before it was placed between two gold-plated brass electrodes of diameter $20 \mathrm{~mm}$. The sample thickness was for all measurements $0.1 \mathrm{~mm}$ as determined by silica spacer fibres.

The TA Instrument DSC Q1000 was used to study thermal events in our samples, i.e., glass transitions, crystallizations, and melting events. The samples (3-25 mg) were placed in hermetically sealed aluminum pans and cooled from $313 \mathrm{~K}$ to $93 \mathrm{~K}$ at a rate of $-30 \mathrm{~K} \mathrm{~min}^{-1}$, before the actual measurements were performed during reheating at a rate of $10 \mathrm{~K} \mathrm{~min}^{-1}$. The glass transition temperature of each sample was determined as the onset of the step in heat capacity.

\section{RESULTS AND DISCUSSION}

\section{A. Differential scanning calorimetry measurements}

The calorimetric glass transition for xylitol and its mixtures with water are shown in Fig. 1. The $T_{g}$ of pure xylitol is located at $247 \mathrm{~K}$, which is in good agreement with previous studies. ${ }^{23,24}$ With increasing water concentration $\mathrm{T}_{\mathrm{g}}$ decreases steadily until the water content is sufficiently high ( $\geq 37$ wt. \%) to cause ice formation already during the cooling procedure (not shown) of the samples. At intermediate water concentrations (29-35 wt. \%) there is no crystallization during the cooling scan, such that $\mathrm{T}_{\mathrm{g}}$ continuous to decrease down to $181 \mathrm{~K}$ (see Fig. 2 for the concentration dependence of $\mathrm{T}_{\mathrm{g}}$ ). In the heating scan almost simultaneous crystallization and melting events occur around $230-240 \mathrm{~K}$ (denoted process 2 in Figs. 1 and 2). The amount of water that crystallize and thereafter melt increases dramatically in the concentration range from 29 to $37 \mathrm{wt}$. \% water, and the two events become also more separated in temperature. At $37 \mathrm{wt}$. \% water two glass transition temperatures are actually observed, the lower one is located at $181 \mathrm{~K}$, i.e., close to the $\mathrm{T}_{\mathrm{g}}$ of the sample with 35 wt. \% water, whereas the higher $\mathrm{T}_{\mathrm{g}}$ is located at about $200 \mathrm{~K}$, see also Fig. 2. The value of the higher $\mathrm{T}_{\mathrm{g}}$ is observed also for a sample of $20 \mathrm{wt}$. \% water, as shown in Fig. 2, as 


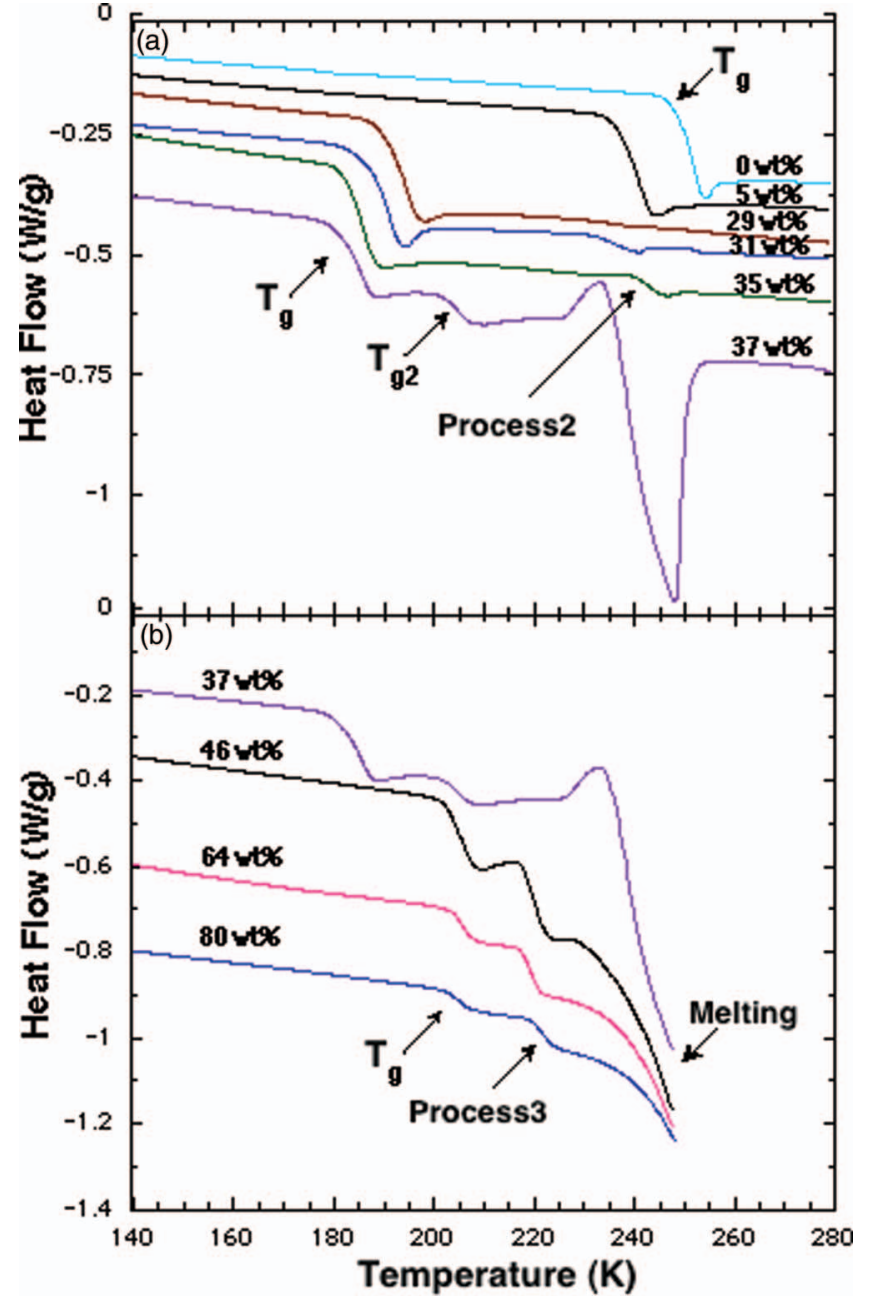

FIG. 1. DSC heating scans of xylitol-water mixtures at different weight fractions of water. (a) Low water concentrations up to 37 wt. \%. (b) High water concentrations from 37 wt. $\%$ up to 80 wt. $\%$. $\mathrm{T}_{\mathrm{g}}$ denotes the glass transition temperature, processes 2 is due to almost simultaneous crystallization and melting events, and process 3 arises from dissolution of ice. The curves have been vertically shifted for clarity $(0 \mathrm{wt}$. $\%$ no shift, $5 \mathrm{wt}$. $\%$ shifted by $-0.03 \mathrm{~W} / \mathrm{g}, 29$ wt. $\%$ shifted by $-0.02 \mathrm{~W} / \mathrm{g}, 31 \mathrm{wt}$. $\%$ shifted by $-0.12 \mathrm{~W} / \mathrm{g}$, 35 wt. $\%$ shifted by $-0.09 \mathrm{~W} / \mathrm{g}, 37$ wt. $\%$ shifted by $-0.19 \mathrm{~W} / \mathrm{g}, 46$ wt. $\%$ shifted by $-0.09 \mathrm{~W} / \mathrm{g}, 64 \mathrm{wt}$ \% shifted by $-0.29 \mathrm{~W} / \mathrm{g}, 80 \mathrm{wt}$ \% shifted by $-0.55 \mathrm{~W} / \mathrm{g})$.

well as for all the samples of higher water content, i.e., for all the samples which undergo partial crystallization already during the cooling scan. This $T_{g}$ reflects the glass transition of a maximally freeze-concentrated solution, which then contains 20 wt. $\%$ water. Thus, for all the samples with more than $37 \mathrm{wt} . \%$ water the unfrozen solution contains $20 \mathrm{wt}$. \% water, and the remaining water is located in ice particles. The presence of two $T_{g}$ for the solution with $37 \mathrm{wt}$. \% water then indicates that one macroscopic part of the sample is unfrozen and has a water concentration close to these $37 \mathrm{wt}$. \%, whereas the other part of the sample is partially crystalline with an effective concentration of unfrozen water of about $20 \mathrm{wt}$. \%. This observation further implies that the concentration of $37 \mathrm{wt} . \%$ water is really the upper limit for which crystallization can be avoided at a cooling rate of $30 \mathrm{~K} \mathrm{~min}^{-1}$.

At the highest water concentrations, above $37 \mathrm{wt} . \%$, we can see in Fig. 1(b) that there is another glass-transition-like

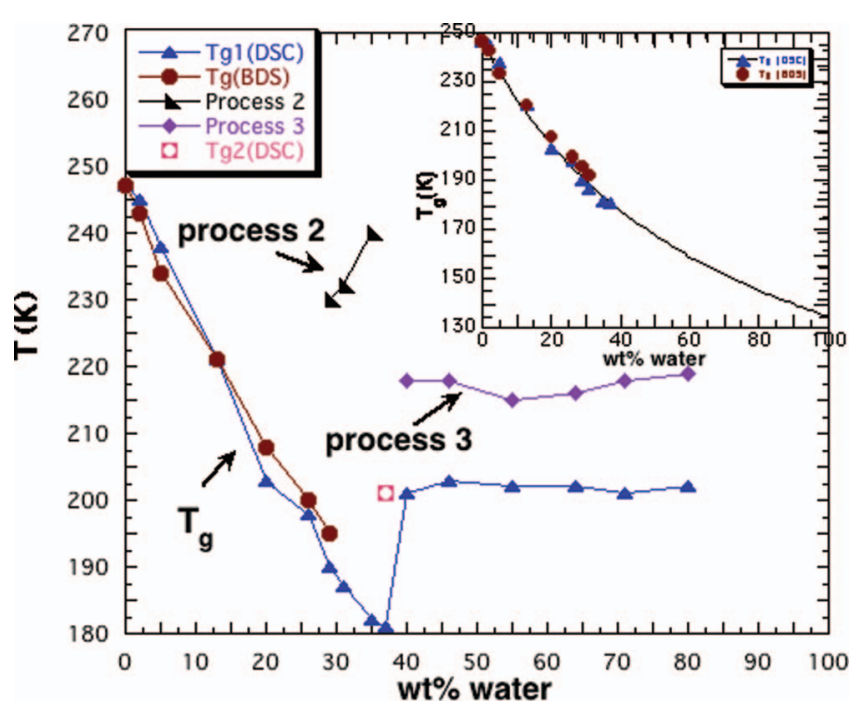

FIG. 2. Concentration dependences of the glass transition temperature, as obtained from DSC and BDS, and the two calorimetric processes 2 and 3 due to almost simultaneous crystallization and melting events and ice dissociation, respectively. The inset shows a Gordon-Taylor fit to the concentration dependence of the glass transition temperature in the concentration range where no ice is formed.

feature at about $220 \mathrm{~K}$ (denoted process 3 in Fig. 2). However, from the careful analysis and discussion made by Inaba and Andersson in Ref. 16 it is most likely that this calorimetric feature is not due to a glass transition, but rather due to dissolution of ice. As for the samples with 29-35 wt. \% water, where no crystallization occur during the cooling scan, cold crystallization occurs near $220 \mathrm{~K}$. Thus, the total amount of ice increases further from the amount produced during the cooling scan. However, upon further heating this supplementary ice will no longer be stable due to that dissolution is energetically more favourable. The reason for this is that the ice particles formed during the cold crystallization will melt according to the extrapolated equilibrium melting line of the xylitol-water system.

In Fig. 2 the calorimetric glass transition temperature is also compared with the dynamic glass transition, i.e., the temperature where the viscosity related structural $(\alpha)$ relaxation reaches a time scale of $100 \mathrm{~s}^{25}$ as obtained by BDS (see below). Comparisons can be made for solutions with water concentrations up to $29 \mathrm{wt}$. \%, where good agreements between the two differently obtained $\mathrm{T}_{\mathrm{g}}$-values can be found, as also shown in Table I. At higher water contents a substantial amount of ice is formed in the dielectric measurements, and comparisons with calorimetric $\mathrm{T}_{\mathrm{g}}$ can therefore not be made. The inset of Fig. 2 shows a Gordon-Taylor fit to the concentration dependence of the glass transition temperature in the concentration range where no ice is formed. Possible implications of this fit are further discussed below.

\section{B. Broadband dielectric spectroscopy measurements}

The imaginary part of the permittivity as a function of frequency is shown in Fig. 3 for the sample containing 20 wt. \% water. At the lowest temperatures up to $190 \mathrm{~K}$ only one dielectric loss peak, due to a relaxation process, can be observed. More exactly this loss peak is caused by the 
TABLE I. Shape parameters a and $\mathrm{b}$ from the Havriliak-Negami function used to describe the structural $\alpha$ relaxation of xylitol-water mixtures. The values are averaged over the whole temperature range where the $\alpha$ relaxation could be clearly observed. Also shown are the glass transition temperatures determined from BDS and DSC, as well as the fragility index $\mathrm{m}$.

\begin{tabular}{lccccc}
\hline \hline & Shape parameter a & Shape parameter b & Fragility index m & $\mathrm{T}_{\mathrm{g}}$ (BDS) K & $\mathrm{T}_{\mathrm{g}}$ (DSC) K \\
\hline Xylitol & $0.93 \pm 0.04$ & $0.30 \pm 0.05$ & 87 & 247 & 247 \\
Xylitol 2 wt. \% $\mathrm{H}_{2} \mathrm{O}$ & $0.92 \pm 0.06$ & $0.29 \pm 0.05$ & 81 & 243 & 245 \\
Xylitol 5 wt. \% $\mathrm{H}_{2} \mathrm{O}$ & $0.78 \pm 0.02$ & $0.37 \pm 0.03$ & 78 & 234 & 238 \\
Xylitol 13 wt. \% $\mathrm{H}_{2} \mathrm{O}$ & $0.66-0.05$ & $0.42-0.04$ & 76 & 221 & 221 \\
Xylitol 20 wt. \% $\mathrm{H}_{2} \mathrm{O}$ & $0.53 \pm 0.05$ & $0.44 \pm 0.06$ & 72 & 208 & 203 \\
Xylitol 26 wt. \% $\mathrm{H}_{2} \mathrm{O}$ & $0.38 \pm 0.03$ & $0.85 \pm 0.06$ & 78 & 200 & 198 \\
Xylitol 29 wt. \% $\mathrm{H}_{2} \mathrm{O}$ & $0.46-0.05$ & $0.72 \pm 0.06$ & 82 & 195 & 190 \\
Xylitol 31 wt. \% $\mathrm{H}_{2} \mathrm{O}$ & $0.54 \pm 0.06$ & $0.82 \pm 0.08$ & $\cdots$ & $\cdots$ & 187 \\
\hline \hline
\end{tabular}

water (w) relaxation mentioned above, in accordance with previous studies of aqueous solutions. ${ }^{8-12}$ With increasing temperature a second loss peak moves into the experimental frequency window at the same time as the w-relaxation moves to higher frequencies. This second peak is due to the structural $\alpha$-relaxation. In Fig. 3 it is also evident that the temperature dependence of the $\alpha$-relaxation is much stronger than for the w-relaxation at low temperatures, and therefore the two processes merge at about $230 \mathrm{~K}$, where only one broad relaxation process can be clearly observed. At these high temperatures also conductivity and polarization (evident from the real part of the permittivity, not shown) effects are giving strong contributions to the data in the low frequency region.

In Fig. 4 we focus on the concentration dependence of the w-relaxation, by showing the imaginary part of the permittivity as a function of frequency for samples of different water contents at a given temperature of $190 \mathrm{~K}$. However, due to the presence of an intrinsic secondary $(\beta)$ relaxation of pure xylitol at almost exactly the same frequency as the w-relaxation it is not possible to determine whether the observed loss peak at low water contents should be considered as the $\beta$-relaxation of xylitol or as a relaxation process caused by the added

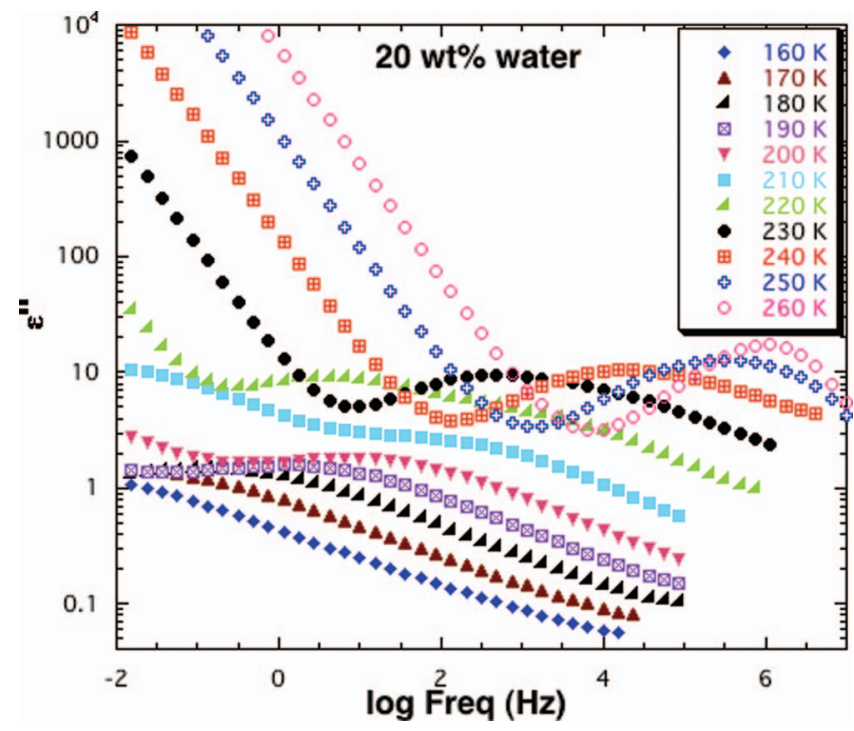

FIG. 3. Dielectric loss spectra of glass forming xylitol with $20 \mathrm{wt}$ \% water at selected temperatures given in the figure. water. Thus, the exact "crossover concentration" from a xylitol dominated process to a water dominated cannot be fully established. An indication of that this "crossover" occurs at a very low water concentration is, however, obtained from the observation that the intensity of the loss peak does not increase with increasing water content at the lowest water concentrations. This is clearly seen in Fig. 4, which shows that the intensity of the secondary relaxation is lowest when 2 wt. \% water has been added to xylitol. Thus, a small quantity of added water seems to efficiently suppress the intrinsic $\beta$-relaxation of xylitol, as previously also has been observed for small quantities of water added to tripropylene glycol. ${ }^{26}$ It is not until the water concentration has increased to $5 \mathrm{wt}$. $\%$ as this secondary relaxation has become as strong as the $\beta$-relaxation of pure xylitol. This observation suggests that the contribution from xylitol to this process decreases rapidly with increasing water content, and that the process is dominated by water even at such a low water concentration as 5 wt. \%. However, it should be noted that this assumed water relaxation at a water content of about $5 \mathrm{wt} . \%$ is

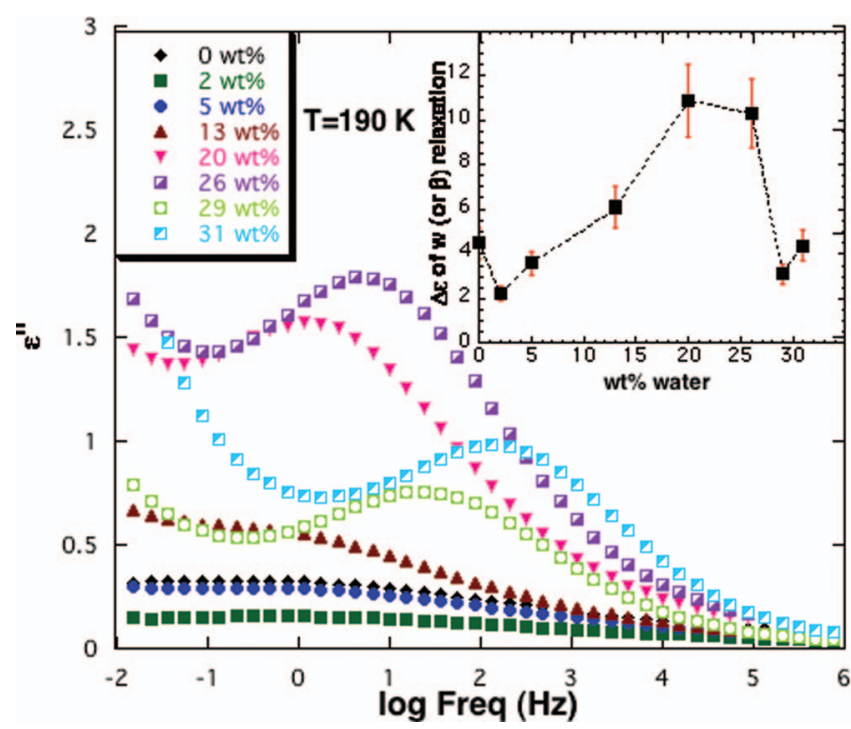

FIG. 4. Dielectric loss spectra of xylitol and xylitol-water mixtures of different water contents, at $\mathrm{T}=190 \mathrm{~K}$. The weight fraction of water in each sample is given in the figure. The inset shows how the intensity of the w (or $\beta$ ) relaxation changes with the water concentration. Partial crystallization is responsible for the decrease in intensity at concentrations above $20 \mathrm{wt} . \%$. 
considerably more similar (similar peak shape, relaxation time, and activation energy) to the intrinsic $\beta$-relaxation of pure xylitol than to the typical relaxation of water in confinements or solutions of high water concentrations. ${ }^{12}$ This indicates that the w-relaxation is, at least partly, caused by the same underlying dynamics as is responsible for the intrinsic $\beta$-relaxation of xylitol. At higher water concentrations the intensity of the w-relaxation increases rapidly with increasing water content up to about 26 wt. $\%$ water, where it starts to decrease. The reason for this decrease is probably that ice particles start to form at these relatively high water contents (29 and 31 wt. \%), as indicated by the almost simultaneous crystallization and melting events on heating in the DSC measurements. However, despite that ice particles most likely have been formed at these water concentrations the w-process continues to speed-up (i.e., shift to higher frequencies) with increasing water content. The reason for this speeding-up of the w-relaxation, despite that the amount of unfrozen water has decreased, is likely that the water molecules interacting with the ice particles exhibit a faster relaxation than water molecules mainly or partly interacting with xylitol molecules. However, as will be shown below, also the $\alpha$-relaxation continues to speed-up with increasing water content up to $31 \mathrm{wt}$. $\%$ water, which suggests that the amount of ice is rather low at this water concentration, in contrast to water contents above 37 wt. \% for which the calorimetric $\mathrm{T}_{\mathrm{g}}$ increases dramatically when ice is formed during the cooling scan, as shown above.

We use standard fit functions to describe the spectra: a power law for the dc-conductivity, a Havriliak-Negami function ${ }^{27}$ for the $\alpha$-process and a Cole-Cole function ${ }^{28}$ for the w-relaxation, as shown in Fig. 5. Thus, for each sample and temperature the total fit function is given by

$$
\varepsilon^{\prime \prime}(\omega)=\frac{\sigma}{\mathrm{i} \varepsilon_{0} \omega}+\frac{\Delta \varepsilon_{\alpha}}{\left(1+\left(\mathrm{i} \omega \tau_{\alpha}\right)^{\mathrm{a}}\right)^{\mathrm{b}}}+\frac{\Delta \varepsilon_{\mathrm{w}}}{1+\left(\mathrm{i} \omega \tau_{\mathrm{w}}\right)^{\mathrm{a}}},
$$

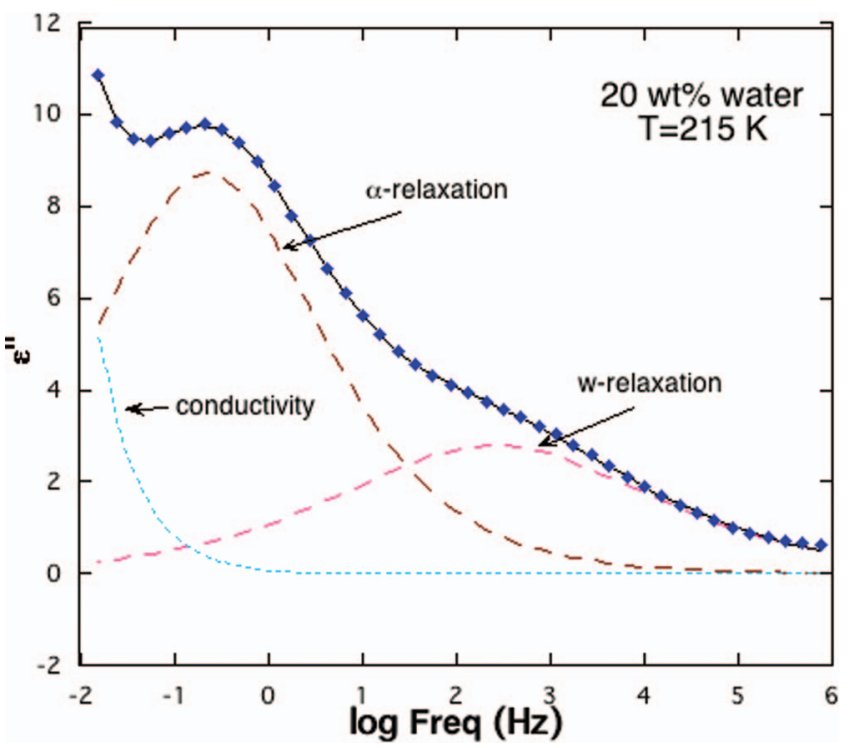

FIG. 5. Dielectric loss spectra of glass forming xylitol with $20 \mathrm{wt}$ \% water at $215 \mathrm{~K}$. The solid line is the resulting fit to the experimental data, using Eq. (1). See the main text for further details about the fitting procedure.
TABLE II. Some fit parameters for the fast $\mathrm{w}$ (or $\beta$ ) relaxation of xylitolwater mixtures. The shape parameter a is from the Cole-Cole function and $\tau_{0}$ and $\mathrm{E}_{\mathrm{a}}$ are from the Arrhenius equation describing the temperature dependence of the relaxation time.

\begin{tabular}{lccc}
\hline \hline & $\tau_{0}(\mathrm{~s})$ & Shape parameter a & $\mathrm{E}_{\mathrm{a}}(\mathrm{kJ} / \mathrm{mol})$ \\
\hline Xylitol & $10^{-20}$ & $0.17 \pm 0.02$ & 64 \\
Xylitol 2 wt. \% $\mathrm{H}_{2} \mathrm{O}$ & $10^{-20}$ & $0.17 \pm 0.03$ & 64 \\
Xylitol 5 wt. \% $\mathrm{H}_{2} \mathrm{O}$ & $10^{-20}$ & $0.19 \pm 0.02$ & 69 \\
Xylitol 13 wt. \% $\mathrm{H}_{2} \mathrm{O}$ & $10^{-22}$ & $0.23 \pm 0.03$ & 74 \\
Xylitol 20 wt. \% $\mathrm{H}_{2} \mathrm{O}$ & $10^{-20}$ & $0.34 \pm 0.03$ & 68 \\
Xylitol 26 wt. \% $\mathrm{H}_{2} \mathrm{O}$ & $10^{-19}$ & $0.38 \pm 0.02$ & 62 \\
Xylitol 29 wt. \% $\mathrm{H}_{2} \mathrm{O}$ & $10^{-19}$ & $0.44 \pm 0.03$ & 59 \\
Xylitol 31 wt. \% $\mathrm{H}_{2} \mathrm{O}$ & $10^{-16}$ & $0.48 \pm 0.03$ & 47 \\
\hline \hline
\end{tabular}

where $\omega=2 \pi \mathrm{f}$ is the angular frequency, $\sigma$ is the dc conductivity, $\tau_{\alpha}$ and $\tau_{\mathrm{w}}$ are the relaxation times for the $\alpha$ and $\mathrm{w}$ (or $\beta$ ) relaxation, respectively, $\Delta \varepsilon_{\mathrm{a}}$ and $\Delta \varepsilon_{\mathrm{w}}$ are the dielectric strengths of the same processes, and a and $b$ are the shape parameters that determine the symmetric and asymmetric broadening of the relaxation peaks, respectively. Some fit parameters for the $\alpha$ and $\mathrm{w}$ (or $\beta$ ) relaxations are given in Tables I and II, respectively. In Table I it can be seen that the shape parameters $\mathrm{a}$ and $\mathrm{b}$ change substantially with increasing water concentration. However, as seen in Fig. 6, where the peak frequency and peak intensity of the $\alpha$-relaxation have been normalized to the same value for all water concentrations, the difference in shape of the of the $\alpha$-relaxation is not as dramatic as the changes of the shape parameters may indicate. The substantial increase of the symmetric broadening (decrease of a) with increasing water content is to some extent compensated by a decrease of the asymmetric broadening (increase of b) on the high frequency side of the peak. The apparent increase of the symmetric broadening may, on the low frequency side of the peak, partly be caused by an increasing contribution from conductivity with increasing water content.

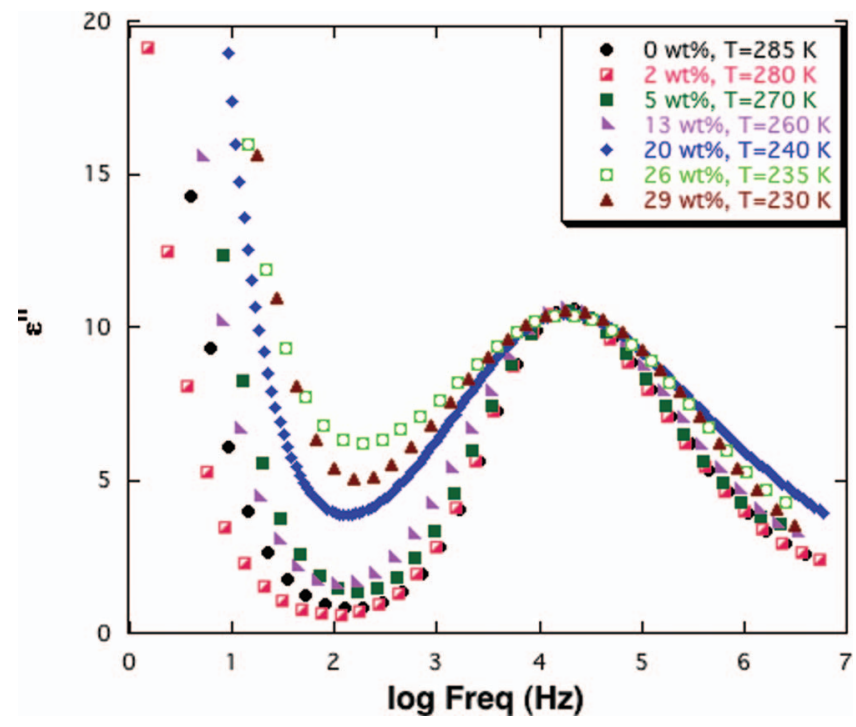

FIG. 6. Concentration dependence of the shape of the structural $(\alpha)$ relaxation. All dielectric loss peaks have been normalized to the same peak intensity and peak frequency as the loss peak of the sample with $20 \mathrm{wt}$. \% water. 


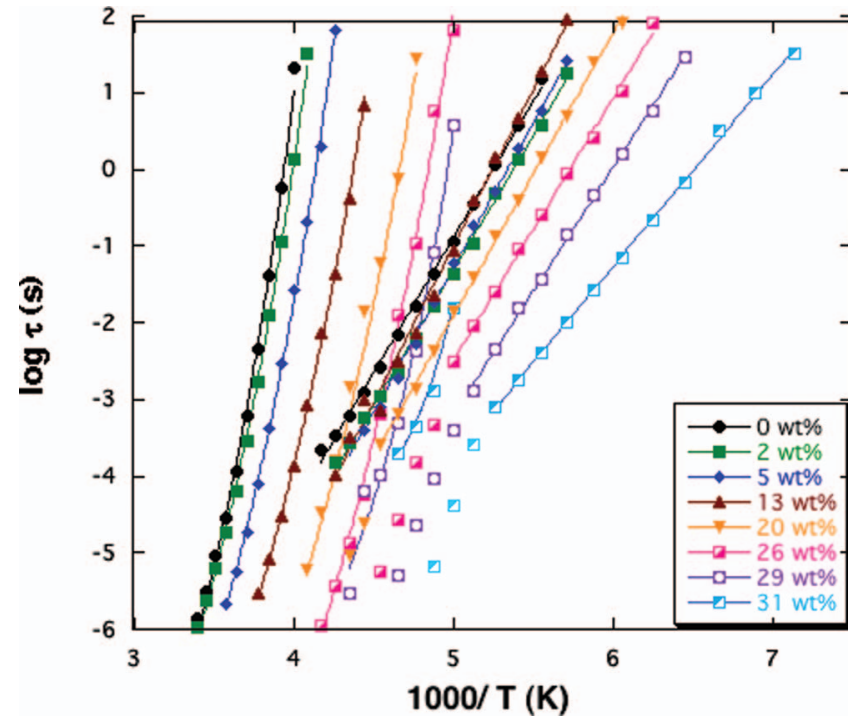

FIG. 7. Temperature dependences of the relaxation processes obtained for xylitol-water mixtures with weight fractions of water as given in the figure. The low temperature dependence of the $\mathrm{w}$ (or $\beta$ ) relaxation time has been fitted by the Arrhenius equation (Eq. (3)), while the VFT equation (Eq. (2)) was used to describe the temperature dependence of the $\alpha$-relaxation time. The fits are given by solid lines. Note that the temperature dependence of the observed $\mathrm{w}$ (or $\beta$ ) relaxation time increases substantially at a temperature close to the merging with the cooperative $\alpha$-relaxation. Possible reasons for this change of activation energy are discussed in the text.

From the curve fitting procedure we obtain the temperature dependent relaxation times of the $\alpha$ and $\mathrm{w}$ (or $\beta$ ) relaxations, as shown in Fig. 7. As usual, the $\alpha$ and $\mathrm{w}$ (or $\beta$ ) relaxations can be distinguished by their temperature dependences. The viscosity related $\alpha$-relaxation shows a nonArrhenius type temperature dependence that is well described by the Vogel-Fulcher-Tammann (VFT) function: ${ }^{29-31}$

$$
\tau_{\alpha}=\tau_{0} \exp \left(\frac{\mathrm{DT}_{0}}{\mathrm{~T}-\mathrm{T}_{0}}\right),
$$

where $\tau_{0}$ is the relaxation time extrapolated to infinite temperature, $T_{0}$ is the temperature where the relaxation time $\tau$ extrapolates to infinity. The parameter $\mathrm{D}$ determines the deviation from Arrhenius temperature dependence, and is related to the fragility of the glass forming liquid. Another estimate of the fragility of the solutions is obtained by calculating the fragility index $\mathrm{m}$, defined as

$$
\mathrm{m}=\left.\frac{\mathrm{d}\left(\log \tau_{\alpha}\right)}{\mathrm{d}\left(\mathrm{T}_{\mathrm{g}} / \mathrm{T}\right)}\right|_{\mathrm{T}=\mathrm{T}_{\mathrm{g}}, 100 \mathrm{~s}},
$$

which measures how rapid $\tau_{\alpha}$ changes with temperature at the dynamics glass transition temperature. In Table I it is shown that the fragility index decreases with increasing water concentration as long as no ice is formed, i.e., up to about $26 \mathrm{wt}$. $\%$ water. Thus, the addition of water to xylitol makes the supercooled liquid less fragile, in accordance to previous studies of similar hydrogen bonded aqueous solutions, $5,22,32,33$ likely due to the increased concentration of hydrogen bonds, which give rise to a network-like structure close to $T_{g}$.

The $\mathrm{w}$ (or $\beta$ ) relaxation is only observed in the deeply supercooled and glassy regimes where it is located at a higher frequency than the structural $\alpha$-relaxation. Its temperature dependence is given by the Arrhenius equation:

$$
\tau_{\beta}=\tau_{0} \exp \left(\frac{\mathrm{E}_{\mathrm{a}}}{\mathrm{k}_{\mathrm{B}} \mathrm{T}}\right),
$$

where $E_{a}$ is the activation energy of the relaxation process. The values we obtain for $\tau_{0}$ and $E_{a}$ are given in Table II. It should here be noted that at water concentrations up to at least 20 wt. \% the activation energy of the w-process is unusually high, compared to most water containing systems (see, e.g., Ref. 12). The reason for this is probably the relatively high activation energy of the intrinsic $\beta$-relaxation of xylitol in combination with a likely coupling between the w-relaxation and the $\beta$-relaxation in this system, at least at water concentrations up to $13 \mathrm{wt}$. \% for which also the time scale of the two processes is almost the same, as shown in Fig. 7. Thus, it seems as the w-relaxation to a large extent is affected by the intrinsic $\beta$-relaxation of xylitol at low water concentrations.

In Fig. 7 it is seen that both the $\alpha$-relaxation and the $\mathrm{w}$ (or $\beta$ ) relaxation become faster with increasing water concentration, except at low water contents $(\leq 13 \mathrm{wt}$. \%) where the $\mathrm{w}$ ( or $\beta$ ) relaxation is basically independent of the water concentration. In addition to the speeding up of the w-relaxation at high water contents its activation energy at low temperatures decreases substantially and the process becomes considerably narrower in frequency (i.e., the fit parameter a increases with increasing water content), as seen in Table II. Note also that the temperature dependence of the observed $\mathrm{w}$ (or $\beta$ ) relaxation time increases substantially at a temperature close to the merging with the cooperative $\alpha$-relaxation. Such increase in activation energy is commonly observed when the time scale of a secondary relaxation process approached that of the $\alpha$-relaxation, which typically occurs around the glass transition temperature of the glass forming material. However, the physical origin of this observed change in activation energy has been a matter of discussion. ${ }^{34-40}$ It can, of course, be explained by a change in relaxation mechanism and associated activation energy at $\mathrm{T}_{\mathrm{g}},{ }^{38}$ but it can in our case be equally well explained by Williams ansatz, ${ }^{39}$ where it is mathematically shown that the observed effective $\beta$-relaxation changes when its time scale approaches that of the $\alpha$-relaxation, even if the activation energy of the true $\beta$-relaxation remains the same above $\mathrm{T}_{\mathrm{g}} \cdot{ }^{40}$ Thus, the merging scenario gives rise to the observed change in activation energy of the effective $\mathrm{w}$ (or $\beta$ ) relaxation even if the relaxation mechanism of the true $\mathrm{w}$ (or $\beta$ ) relaxation remains the same above $\mathrm{T}_{\mathrm{g}}$.

In contrast to the observations in Ref. 5 , where only solutions with water contents up to $10 \mathrm{wt} . \%$ was studied, our results show that the intrinsic $\beta$-relaxation of xylitol is considerably broader in frequency than the w-relaxation at higher water contents, but also that the water molecules mainly interacting with xylitol (as is the case at low water contents) are significantly less mobile than water molecules mainly interacting with other water molecules (as is the case at the higher water contents). The fact that ice clusters start to form at a water concentration of about $30 \mathrm{wt}$. \% is, indeed, a good proof that water clusters of substantial sizes are formed at such concentrations. In hard cylindrical confinements ice formation is avoided for pore sizes as large as $21 \AA$, ${ }^{41}$ which implies that relatively large water clusters have to be formed before 
the nucleation process starts. Thus, it is clear that the typical local environment of a water molecule changes drastically from being "isolated" in a network of hydrogen bonded xylitol molecules, and therefore governed by the intrinsic $\beta$-relaxation of xylitol at very low water contents, to participating in a network of mainly surrounding water molecules, at concentrations close to $30 \mathrm{wt}$. $\%$ water, and that this change in local environment is reflected as a decrease in activation energy and a narrowing and speeding up of the water relaxation.

As mentioned above, the dynamic glass transition temperature can be determined from the temperature dependent $\alpha$-relaxation as the temperature where this process reaches a time scale of $100 \mathrm{~s}^{25}$ The so obtained $\mathrm{T}_{\mathrm{g}}$-values are compared with the calorimetrically obtained $\mathrm{T}_{\mathrm{g}}$-values in Fig. 2 and Table I. The two differently obtained $\mathrm{T}_{\mathrm{g}}$-values are in good agreement and shown to rapidly decrease with increasing water content in the concentration range up to about 30 wt. \% water where ice formation can be avoided even in the BDS measurements. This implies that water acts as a plasticizer for xylitol, which has been shown to be the most common effect water has on other hydrogen bonding glass forming liquids. ${ }^{6,9,11,18,42}$ However, in cases when added water molecules participate in the build-up of hydrogen bonded supramolecular structures an opposite scenario can be observed and $\mathrm{T}_{\mathrm{g}}$ increases with increasing water content, up to a certain water concentration. ${ }^{10,19,21}$ Since this is not the case for xylitol-water mixtures the results indicate that a hydrogen bonding network is formed in pure xylitol as well as in the water containing samples. However, from a dynamic point of view it is important to note that $\mathrm{H}$-bonds are relatively weak and only stable on a time-scale of a ps. ${ }^{43}$ This implies that networks of hydrogen bonded molecules are constantly changing and allowing the molecules to relax through the structural $\alpha$ relaxation and one or several more local relaxation processes. In the present xylitol-water system it is clear that the added water molecules increase the network character, and thereby also decrease the fragility, of the supercooled liquid, but, nevertheless, the lower molecular weight of the water molecules, compared to the xylitol molecules, makes the network of $\mathrm{H}$-bonded molecules more flexible and rapidly changing at high water concentrations. This not only speeds up the viscosity related $\alpha$-relaxation at a given temperature but also produces a more local and specific water relaxation, which decouples from the $\alpha$-relaxation close to $\mathrm{T}_{\mathrm{g}}$.

If $\mathrm{T}_{\mathrm{g}}$ is extrapolated to pure water by the Gordon-Taylor equation $^{16,44}$ a value of about $135 \mathrm{~K}$ is obtained, see the inset of Fig. 2. Although the Gordon-Taylor equation is not accurately describing the concentration dependent glass transition temperature of all aqueous solutions, ${ }^{45}$ it describes the $\mathrm{T}_{\mathrm{g}}$ variation of the present xylitol-water mixtures to a good accuracy in the available (i.e., unfrozen) concentration range. Therefore, it may not be surprising that this possible $\mathrm{T}_{\mathrm{g}}$-value of bulk water is in excellent agreement with the most acceptable value of $136 \mathrm{~K}$ found in the literature. ${ }^{46} \mathrm{~A}$ similar support for a $\mathrm{T}_{\mathrm{g}}$-value around $136 \mathrm{~K}$ for bulk water was obtained by Cappaccioli and $\mathrm{Ngai}^{47}$ in a recent study of a wide range of water containing systems. They also reached the conclusion that the Johari-Goldstein $\beta$-relaxation of water (denoted
W-relaxation in this paper) is close in relaxation time to the $\alpha$-relaxation at $T_{g}$ and merges with it just above $T_{g}$. Since the here observed w-relaxation in the sample of highest water concentration ( $31 \mathrm{wt} . \%)$ is reaching a relaxation time of $100 \mathrm{~s}$ at about $136 \mathrm{~K}$ our results support their idea ${ }^{40}$ that $\alpha$ and $\beta$ of bulk water may be close in relaxation time at $\mathrm{T}_{\mathrm{g}}$. It should, however, be noted that a slightly lower "freezingin temperature" of the w-relaxation had probably been observed if substantial crystallization could be avoided in samples of higher water concentrations, as also indicated for the w-relaxation in a wide range of confinements. ${ }^{41,48-52}$ The similarity of the $T_{g}$ extrapolated to pure water and the "freezing-in temperature" of the w-relaxation furthermore suggests that the $\alpha$-relaxation of the xylitol-water solution extrapolates to the w-relaxation with increasing water content. However, as discussed in Ref. 53 and also indicated in this study, this w-relaxation should not be considered to be a viscosity related $\alpha$-relaxation, but a more local secondary relaxation process of possibly the Johari-Goldstein type. ${ }^{54}$ Therefore, it is still difficult to establish whether the assumed glass transition that has been experimentally observed at about $136 \mathrm{~K}^{46}$ for bulk water should be considered as a true $\mathrm{T}_{\mathrm{g}}$ of bulk water or be caused by the "freezing-in" of the more local w-relaxation, as previously has been suggested. ${ }^{55}$

\section{CONCLUSIONS}

The DSC measurements showed a complex calorimetric behaviour of xylitol-water mixtures at water concentrations above 29 wt. \%, for which different types of crystallization and melting events occurred in addition to the glass transition. The glass transition temperature decreased steadily with increasing water content up to $37 \mathrm{wt}$. \% water, where partial crystallization of the water occurs already during the precooling of the samples. At higher water contents a constant $\mathrm{T}_{\mathrm{g}}$ of about $200 \mathrm{~K}$ was obtained, corresponding to a maximally freeze-concentrated solution of $20 \mathrm{wt}$. \% water. In this concentration range, where ice was formed during both the pre-cooling and the heating of the samples, another glasstransition-like feature was observed at about $220 \mathrm{~K}$, but this feature is most likely due to ice dissolution, since the ice particles formed during the cold crystallization will no longer be energetically favourable and therefore melt according to the extrapolated equilibrium melting line of the xylitol-water system.

The dielectric results of xylitol mixed with water show two relaxation processes, the $\alpha$ and $\mathrm{w}$ (or $\beta$ ) relaxation. Both relaxations are strongly dependent on the water concentration of the xylitol-water mixture. The structural $\alpha$-relaxation speeds up with increasing water content simultaneously as the fragility of the supercooled liquid decreases. The decrease in fragility is caused by an increase in the density of hydrogen bonds, which gives rise to a more network-like structure, whereas the speeding up of the $\alpha$-relaxation is likely due to the substantially lower molecular weight of water compared to xylitol.

A small quantity of water added to xylitol seems to suppress the intrinsic $\beta$-relaxation of xylitol, and at water contents above $5 \mathrm{wt}$. $\%$ the intrinsic $\beta$-relaxation is replaced by 
a more intense water relaxation at a similar frequency. This w-relaxation shows an Arrhenius temperature dependence, as typical for local secondary relaxations, and its intensity increases with increasing water concentration up to about 26 wt. \% water. At low water concentrations (up to about 13 wt. \%) it is very similar to the $\beta$-relaxation of xylitol, and therefore most likely governed by the same underlying dynamics as is responsible for the $\beta$-relaxation. At water concentrations above $26 \mathrm{wt}$. $\%$ small ice clusters are probably formed during the dielectric measurements, and therefore the intensity of the w-relaxation decreases.

\section{ACKNOWLEDGMENTS}

This work was financially supported by the Swedish Research Council and the Swedish Energy Agency.

${ }^{1}$ G. H. Hildebrandt and B. S. Sparks, J. Am. Dent. Assoc. 131, 909 (2000).

${ }^{2}$ A. B. Burt, J. Am. Dent. Assoc. 137, 191 (2006).

${ }^{3}$ J. C. Parajó, H. Dominguez, and J. M. Dominguez, Bioresource Tech. 65, 191 (1998).

${ }^{4}$ T. Kontiokaria, M. Uhari, and M. Koskela, J. Antimicrob. Chemother. 41, 563 (1998).

${ }^{5}$ T. Psurek, S. Maslanka, M. Paluch, R. Nozaki, and K. L. Ngai, Phys. Rev. E 70, 011503 (2004).

${ }^{6}$ R. A. Talja and Y. H. Roos, Thermochim. Acta 380, 109 (2001).

${ }^{7}$ G. P. Johari and M. Goldstein, J. Chem. Phys. 53, 2372 (1970).

${ }^{8}$ N. Shinyashiki, S. Sudo, S. Yagihara, A. Spanoudaki, A. Kyritsis, and P. Pissis, J. Phys. Condens. Matter 19, 205113 (2007).

${ }^{9}$ S. Cerveny, G. Schwartz, A. Alegria, R. Bergman, and J. Swenson, J. Chem. Phys. 124, 194501 (2006).

${ }^{10}$ J. Sjöström, J. Mattsson, R. Bergman, E. Johansson, K. Josefsson, D. Svantesson, and J. Swenson, Phys. Chem. Chem. Phys. 12, 10452 (2010).

${ }^{11}$ S. Cerveny, J. Colmenero, and A. Alegria, J. Non-Cryst. Solids 353, 4523 (2007)

${ }^{12}$ S. Cerveny, A. Alegria, and J. Colmenero, Phys. Rev. E 77, 031803 (2008).

${ }^{13}$ S. Sudo, S. Tsubotani, M. Shimomura, N. Shinyashiki, and S. Yagihara, J. Chem. Phys. 121, 7332 (2004).

${ }^{14}$ S. Cerveny, J. Colmenero, and A. Alegria, Macromolecules 38, 7056 (2005).

${ }^{15}$ R. Nozaki, H. Zenitani, A. Minoguchi, and K. Kitai, J. Non-Cryst. Solids 307, 349 (2002).

${ }^{16} \mathrm{~A}$. Inaba and O. Andersson, Thermochim. Acta 461, 44 (2007).

${ }^{17}$ M. Shinohara, T. Goto, M. Oyama, S. Suzuki, N. Shinyashiki, S. Yagihara, T. Inoue, S. Oyaizu, and S. Yamamoto, AIP Conf. Proc. 832, 145 (2006).

${ }^{18} \mathrm{H}$. Jansson, R. Bergman, and J. Swenson, J. Non-Cryst. Solids 351, 2858 (2005).
${ }^{19}$ J. Sjöström, J. Mattsson, R. Bergman, and J. Swenson, J. Phys. Chem. B 115, 10013 (2011).

${ }^{20}$ J. Mattsson, R. Bergman, P. Jacobsson, and L. Börjesson, Phys. Rev. B 79, 174205 (2009).

${ }^{21}$ J. Swenson, J. Sjöström, and F. Fernandez-Alonso, J. Chem. Phys. 133, 234506 (2010)

${ }^{22}$ S. E. Pagnotta, S. Cerveny, A. Alegria, and J. Colmenero, J. Chem. Phys. 131, 085102 (2009).

${ }^{23}$ H. Jansson, R. Bergman, and J. Swenson, J. Mol. Struct. 972, 92 (2010).

${ }^{24}$ R. Bergman, H. Jansson, and J. Swenson, J. Chem. Phys. 132, 044504 (2010).

${ }^{25}$ R. Böhmer, K. L. Ngai, C. A. Angell, and D. J. Plazek, J. Chem. Phys. 99, 4201 (1993).

${ }^{26}$ J. Sjöström, R. Bergman, C. Wadell, T. Moberg, J. Mattsson, and J. Swenson, J. Phys. Chem. B 115, 1842 (2011).

${ }^{27}$ S. Havriliak and S. Negami, Polymer 8, 161 (1967).

${ }^{28}$ K. S. Cole and R. H. Cole, J. Chem. Phys. 9, 341 (1941).

${ }^{29}$ H. Vogel, Phys. Z. 22, 645 (1921).

${ }^{30}$ G. S. Fulcher, J. Am. Ceram. Soc. 8, 339 (1925).

${ }^{31}$ G. Tammann and W. Hesse, Z. Anorg. Allg. Chem. 156, 245 (1926).

${ }^{32}$ C. A. Angell, Chem. Rev. 102, 2627 (2002).

${ }^{33}$ K. Ito, C. T. Moynihan, and C. A. Angel, Nature (London) 398, 492 (1999).

${ }^{34}$ T. Fujima, H. Frusawa, and K. Ito, Phys. Rev. E 66, 031503 (2002).

${ }^{35}$ K. L. Ngai and M. Paluch, J. Chem. Phys. 120, 857 (2004).

${ }^{36}$ F. Alvarez, A. Hoffman, A. Alegria, and J. Colmenero, J. Chem. Phys 105, 432 (1996).

${ }^{37}$ C. Svanberg, R. Bergman, and P. Jacobsson, Europhys. Lett. 64, 358 (2003)

${ }^{38}$ K. L. Ngai and S. Capaccioli, Phys. Rev. E 69, 031501 (2004).

${ }^{39}$ G. Williams, Adv. Polym. Sci. 33, 60 (1979).

${ }^{40}$ R. Bergman and C. Svanberg, Phys. Rev. E 72, 043501 (2005).

${ }^{41}$ J. Hedström, J. Swenson, R. Bergman, H. Jansson, and S. Kittaka, Eur. Phys. J. Spec. Top. 141, 53 (2007).

${ }^{42}$ F. Salaün, G. Bedek, E. Devaux, D. Dupont, and D. Deranton, Int. J. Thermophys. 30, 1242 (2009).

${ }^{43}$ A. Luzar and D. Chandler, Phys. Rev. Lett. 76, 928 (1996).

${ }^{44}$ B. C. Hancock and G. Zografi, Pharm. Res. 11, 471 (1994).

${ }^{45}$ S. S. N. Murthy and G. Singh, Thermochim. Acta 469, 116 (2008).

${ }^{46}$ G. P. Johari, A. Hallbrucker, and E. Mayer, Nature (London) 330, 552 (1987).

${ }^{47}$ S. Capaccioli and K. L. Ngai, J. Chem. Phys. 135, 104504 (2011).

${ }^{48}$ H. Jansson and J. Swenson, Eur. Phys. J. E 12, 51 (2002).

${ }^{49}$ J. Swenson, H. Jansson, W. S. Howells, and S. Longeville, J. Chem. Phys. 122, 084505 (2005)

${ }^{50}$ R. Bergman and J. Swenson, Nature (London) 403, 283 (2000).

${ }^{51}$ R. Bergman, J. Swenson, L. Börjesson, and P. Jacobsson, J. Chem. Phys. 113, 357 (2000)

${ }^{52}$ J. Sjöström, J. Swenson, R. Bergman, and S. Kittaka, J. Chem. Phys. 128, 154503 (2008).

${ }^{53}$ J. Swenson, H. Jansson, and R. Bergman, Phys. Rev. Lett. 96, 247802 (2006).

${ }^{54}$ S. Capaccioli, K. L. Ngai, and N. Shinyashiki, J. Phys. Chem. B 111, 8197 (2007).

${ }^{55}$ J. Swenson and J. Teixeira, J. Chem. Phys. 132, 014508 (2010). 